\title{
Establishing a critical care network in Asia to improve care for critically ill patients in low- and middle-income countries
}

\author{
CRIT CARE ASIA
}

Keywords: Critical care, Quality improvement, Registry, Low- and middle-income countries

\section{Introduction}

When undertaking quality improvement (QI) initiatives, one of the greatest burdens is repeated data collection. Intensive care registries, such as those commonly used in high-income countries (HICs), have enabled systematic capture of routine information needed to measure intensive care unit (ICU) performance [1]. Once considered unfeasible in resource-limited settings, newer cloud-based platforms are gaining increasing traction. Collaborative surveillance platforms, such as NICS-MORU and PRICE, which have mobile and desktop applications, have established methods for daily capture of individual patient-level information and have shown that-even in resourcelimited settings-the systematic evaluation of patient care throughout the hospital journey is feasible at scale using coalesced minimal data sets $[2,3]$.

\section{Quality of care}

Poor quality of care has resulted in an estimated additional five million deaths, and six trillion US dollars in economic losses worldwide [4, 5]. In South and SouthEast Asia, a region which accounts for over $25 \%$ of the world's population, poor quality health care is one of the biggest drivers of excess morbidity and mortality [4]. Recent recommendations from the Lancet Global Health Commission have called for greater investment in systems that strengthen evaluation and improvement, and a focus

\section{Correspondence:}

Collaboration for Research, Implementation and Training in intensive CARE in ASIA, Mahidol Oxford Tropical Research Unit, 3/F, 60th Anniversary

Chalermprakiat Building, 420/6 Rajvithi Road, Bangkok, Thailand on healthcare that is reflective of and sensitive to the diverse needs of communities [5].

Critical care is expensive and complex. Many barriers impede the optimal care of critically ill patients, especially in resource-restricted settings [6]. Basic equipment for monitoring, treatment and diagnosis is often unavailable and maintenance is suboptimal [7]. Supplies of laboratory consumables and essential medications can be unpredictable and the provision of basic commodities, such as oxygen, electricity and running water, unreliable. Despite these challenges, demand for these services continues and with it the need to establish systems by which quality of care can be continually improved.

\section{Barriers to quality improvement in LMICs} Lack of information for quality evaluation

The ability to continually evaluate care and empower stakeholders to identify priorities for improvement is a crucial but missing component of QI $[4,8]$. In low- and middle-income countries (LMICs), the lack of reliable facility-level and national information has hampered the implementation of QI initiatives and prevented clinicians from identifying local research priorities [2].

\section{Limited success of quality improvement}

Many of the basic principles of 'good quality' critical care that have proved successful in HICs may be directly applicable to resource-limited settings. Often, however, practices are poorly implemented. To date, in LMICs, QI initiatives have had limited success in achieving sustained change or have proven difficult to scale [5]. Quality 
improvement methods are generally neither an established part of medical education nor are they a priority investment for healthcare institutions in resource-limited settings $[5,9]$.

\section{A pathway for improving quality of care}

Supported by a Wellcome Innovations Flagship Programme grant, our group of multidisciplinary healthcare professionals predominantly based in Asia, is establishing a locally led collaborative network: Collaboration for Research, Implementation and Training in intensive CARE in ASIA (CRIT CARE ASIA). The collaboration will improve patient outcomes using near real-time high-quality data to drive improvement and strengthen the health system through a system of audit and feedback. Delivered over 3 years and extendable beyond this period, the programme will establish an Asian ICU network across 42 units in nine countries and implement a setting-adapted electronic cloud-based registry co-designed and developed by clinicians in the region (Fig. 1). Using the registry, plus qualitative and quantitative research methods, CRIT CARE ASIA will evaluate the quality of critical care, which will then lead into locally led QI interventions to improve ICU performance and patient outcomes driven by the priorities of stakeholders.

\section{Establishing a collaborative network}

Initiating, maintaining or advancing a local improvement programme consumes large amounts of time and energy [11]. Establishing or joining a collaboration for shared improvement, such as CRIT CARE ASIA, may help. Collaborative improvement networks engage clinicians, researchers, patients and policy-makers to test approaches to improve care, translate research and prioritise service delivery $[2,3]$. CRIT CARE ASIA will connect ICUs and institutions to provide diverse high-quality data using an agreed core dataset to generate evidence and inform clinical decision-making. The network uses collaboration, data science, clinical training and implementation science methods pragmatically adapted to resource-limited settings. These combined methods create a feedback loop within the ICU and allow operational problems to be quickly corrected and potential pitfalls of implementation of the QI intervention to be avoided.

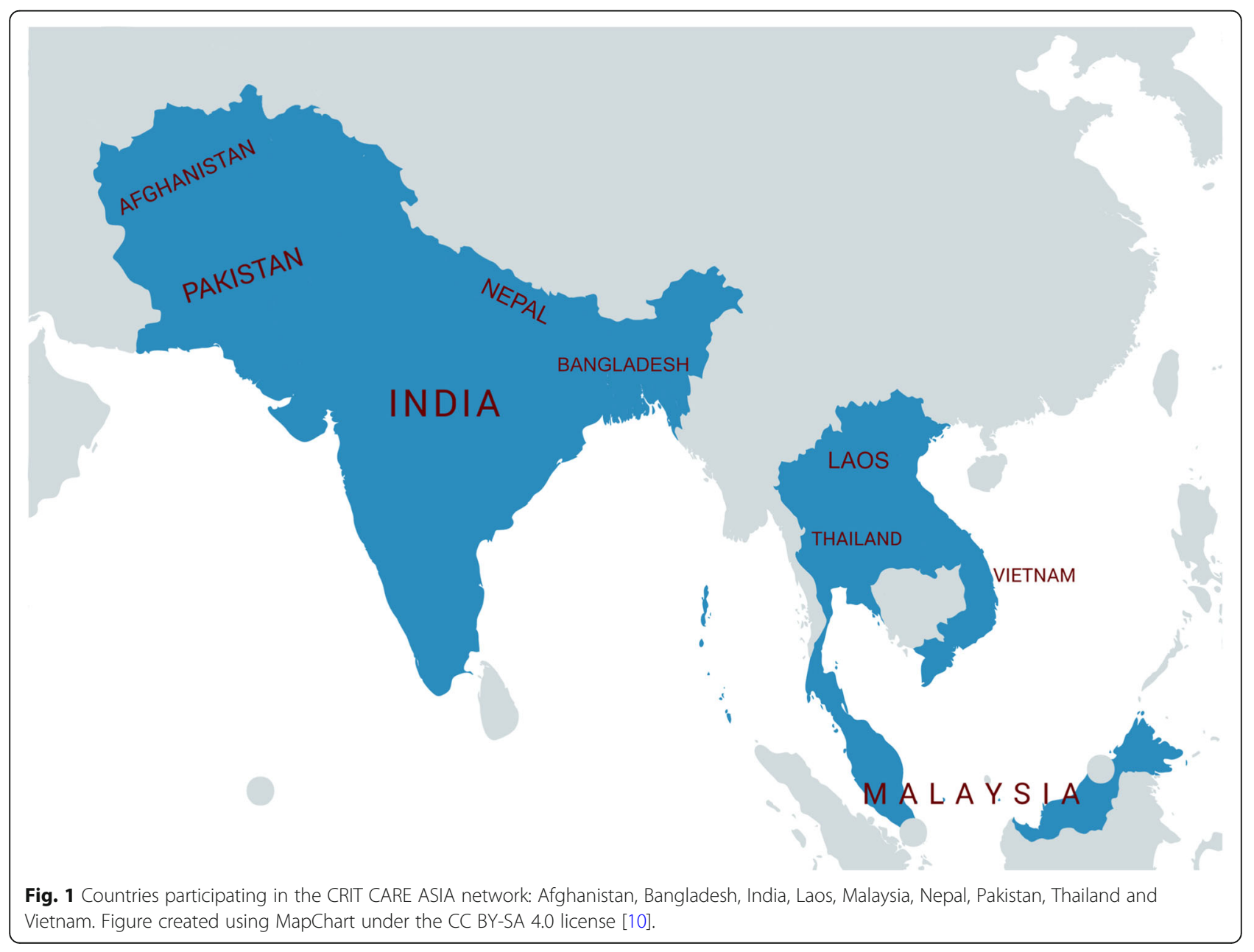




\section{Utilise a problem-solving approach to quality improvement}

To improve quality of care, it is necessary to locate the gap in care and to identify and understand its underlying determinants. Utilising mixed-methods, the network will encourage a learning by doing model, whereby members of the network will be supported to interact with data generated through the registry and identify problems and possible solutions. Qualitative approaches, including 'real-time evaluations', from disciplines including anthropology and business, which lend themselves to rapid evaluation of complex health systems, will be utilised [12]. Findings from interviews, observations and focus group discussions will inform the design and implementation of targeted QI projects.

\section{Challenges}

The greatest challenge lies with information governance aspects of data curation and sharing. The diverse project team will use their extensive LMIC experience to overcome barriers to data sharing. International research partnerships can disproportionately advantage high-income countries and institutions [13]. With this in mind, we have proactively incorporated measures including inclusive priority-setting and locally led improvement into the design of the network activities.

\section{Future}

The network will enable the implementation and evaluation of innovative LMIC technologies and provide opportunity for data linkage with the registry [14]. The ICU network, supported by the electronic registry, will facilitate epidemiological and clinical research. CRIT CARE ASIA uses a Common Data Model and standard nomenclature and coding, which will facilitate comparison of data with globally relevant health data networks such as ISARIC and LOGIC. Many critically unwell patients are cared for outside of the ICU [6]. Many of the strategies to improve the quality of processes of care will therefore need to extend beyond the ICU to encompass surgical, emergency medicine and communitybased services as already demonstrated in Sri Lanka [15].

\section{Abbreviations}

CRIT CARE ASIA: Collaboration for Research, Improvement and Training in Critical CARE in ASIA; HIC: High-income country; ICU: Intensive care unit; LMIC: Low- and middle-income country; QI: Quality improvement

\footnotetext{
Acknowledgements

CRIT CARE ASIA group (listed alphabetically based on first name): Abi Beane, Arjen M. Dondorp, Arshad Taqi, ASM Areef Ahsan, Bharath Kumar Tirupakuzhi Vijayaraghavan, Chairrat Permpikul, Christopher Pell, David Gandy, Dilanthi Priyadarshani, Diptesh Aryal, Dong Phu Khiem, Duong Bich Thuy, Guy Thwaites, Gyan Kayastha, Ishara Udayanga, Jorge Salluh, Khamsay Detleuxay, Lakshmi Ranganathan, Lam Minh Yen, Lim Chew Har, Louise Thwaites, Madiha Hashmi, Marcus J. Schultz, Mavuto Mukaka, Meghan Leaver, Mohd Basri Mat Nor, Muhammad Hayat, Nick Day, Ramani Moonesinghe, Rashan Haniffa, Ratapum Champunot, Rebecca Inglis, Rozina Sultana, Sophie Yacoub,
}

Steve Harris, Subhash Prasad Acharya, Swagata Tripathy, Syed Muneeb Ali, Tamilarasu Kadhiravan and Yoel Lubell.

\section{Authors' contributions}

$A B$ wrote the original draft. All authors reviewed and edited subsequent drafts, and read and approved the final version of the manuscript.

\section{Funding}

This work was undertaken as part of the existing Wellcome Innovations Flagship award, Collaboration for Research, Improvement and Training in Critical CARE in ASIA (ref. 215522/Z/19/Z). The funder had no role in the decision to publish or in the preparation of this manuscript.

\section{Availability of data and materials}

Data sharing is not applicable to this article as no datasets were generated or analysed during the current study.

Ethics approval and consent to participate

Not applicable.

Consent for publication

Not applicable.

\section{Competing interests}

The authors declare that they have no competing interests.

Received: 29 September 2020 Accepted: 1 October 2020

Published online: 15 October 2020

\section{References}

1. van der Veer SN, de Keizer NF, Ravelli ACJ, Tenkink S, Jager KJ. Improving quality of care. A systematic review on how medical registries provide information feedback to health care providers. Int J Med Inform. 2010;79: 305-23.

2. Beane A, De Silva AP, Athapattu PL, Jayasinghe S, Abayadeera AU, Wijerathne $\mathrm{M}$, et al. Addressing the information deficit in global health: lessons from a digital acute care platform in Sri Lanka. BMJ Glob Health. 2019:4:1-7.

3. Hashmi M, Beane A, Taqi A, Memon Ml, Athapattu P, Khan Z, et al. Pakistan Registry of Intensive CarE (PRICE): expanding a lower middle-income, clinician-designed critical care registry in South Asia. Pediatr Crit Care Med. 2019;20:190-5

4. Kruk ME, Gage AD, Joseph NT, Danaei G, García-Saisó S, Salomon JA. Mortality due to low-quality health systems in the universal health coverage era: a systematic analysis of amenable deaths in 137 countries. Lancet. 2018; 392:2203-12.

5. Kruk ME, Gage AD, Arsenault C, Jordan K, Leslie HH, Roder-DeWan S, et al. High-quality health systems in the Sustainable Development Goals era: time for a revolution. Lancet Glob Health. 2018;6:e1196-252.

6. Schell CO, Gerdin Wärnberg M, Hvarfner A, Höög A, Baker U, Castegren M, et al. The global need for essential emergency and critical care. Crit Care. 2018;22:284

7. Arabi YM, Phua J, Koh Y, Du B, Faruq MO, Nishimura M, et al. Structure, organization, and delivery of critical care in Asian ICUs. Crit Care Med. 2016; 44:e940-8.

8. English M, Irimu G, Agweyu A, Gathara D, Oliwa J, Ayieko P, et al. Building learning health systems to accelerate research and improve outcomes of clinical care in low- and middle-income countries. PLOS Medicine. 2016. e1001991. Available from: https://doi.org/10.1371/ journal.pmed.1001991.

9. Beane A, Wagstaff D, Abayadeera A, Wijeyaratne M, Ranasinghe G, Mirando $S$, et al. A learning health systems approach to improving the quality of care for patients in South Asia. Glob Health Action; 2019;12. Available from: https://doi.org/10.1080/16549716.2019.1587893.

10. MapChart: Asia. https://mapchart.net/asia.html. Accessed 8 Oct 2020

11. Vos MLG de, van der Veer SN, Graafmans WC, de Keizer NF, Jager KJ, et al. Process evaluation of a tailored multifaceted feedback program to improve the quality of intensive care by using quality indicators. BMJ Quality Safety. 2013. 233-41. Available from: https://doi.org/10.1136/ bmjqs-2012-001375. 
12. Vindrola-Padros C, Pape T, Utley M, Fulop NJ. The role of embedded research in quality improvement: a narrative review. BMJ Qual Saf. 2017; 26:70-80.

13. World Health Organization, TDR. TDR research fairness initiative report 2018. World health Organization; 2018. Available from: https:/apps.who.int/iris/ bitstream/handle/10665/260339/TDR-STRA-18.1-eng.pdf.

14. Turner HC, Van Hao N, Yacoub S, Hoang VMT, Clifton DA, Thwaites GE, et al. Achieving affordable critical care in low-income and middle-income countries. BMJ Glob Health. 2019;4:1-4.

15. Ranasinghe WG, Beane A, Vithanage TDP, Priyadarshani GDD, Colombage DDE, Ponnamperuma CJ, et al. Quality evaluation and future priorities for delivering acute myocardial infarction care in Sri Lanka. Heart. 2020;106:603-8.

\section{Publisher's Note}

Springer Nature remains neutral with regard to jurisdictional claims in published maps and institutional affiliations. 